ORIGINAL ARTICLE

\section{Atenolol versus Losartan in Children and Young Adults with Marfan's Syndrome}

\author{
R.V. Lacro, H.C. Dietz, L.A. Sleeper, A.T. Yetman, T.J. Bradley, S.D. Colan, \\ G.D. Pearson, E.S. Selamet Tierney, J.C. Levine, A.M. Atz, D.W. Benson, \\ A.C. Braverman, S. Chen, J. De Backer, B.D. Gelb, P.D. Grossfeld, G.L. Klein, \\ W.W. Lai, A. Liou, B.L. Loeys, L.W. Markham, A.K. Olson, S.M. Paridon, \\ V.L. Pemberton, M.E. Pierpont, R.E. Pyeritz, E. Radojewski, M.J. Roman, \\ A.M. Sharkey, M.P. Stylianou, S. Burns Wechsler, L.T. Young, and L. Mahony, \\ for the Pediatric Heart Network Investigators*
}

A BSTRACT

\section{BACKGROUND}

Aortic-root dissection is the leading cause of death in Marfan's syndrome. Studies suggest that with regard to slowing aortic-root enlargement, losartan may be more effective than beta-blockers, the current standard therapy in most centers.

\section{METHODS}

We conducted a randomized trial comparing losartan with atenolol in children and young adults with Marfan's syndrome. The primary outcome was the rate of aorticroot enlargement, expressed as the change in the maximum aortic-root-diameter z score indexed to body-surface area (hereafter, aortic-root z score) over a 3-year period. Secondary outcomes included the rate of change in the absolute diameter of the aortic root; the rate of change in aortic regurgitation; the time to aortic dissection, aortic-root surgery, or death; somatic growth; and the incidence of adverse events.

\section{RESULTS}

From January 2007 through February 2011, a total of 21 clinical centers enrolled 608 participants, 6 months to 25 years of age (mean [ \pm SD] age, $11.5 \pm 6.5$ years in the atenolol group and 11.0 \pm 6.2 years in the losartan group), who had an aorticroot $\mathrm{z}$ score greater than 3.0. The baseline-adjusted rate of change $( \pm \mathrm{SE})$ in the aortic-root $\mathrm{z}$ score did not differ significantly between the atenolol group and the losartan group $(-0.139 \pm 0.013$ and $-0.107 \pm 0.013$ standard-deviation units per year, respectively; $\mathrm{P}=0.08)$. Both slopes were significantly less than zero, indicating a decrease in the degree of aortic-root dilatation relative to body-surface area with either treatment. The 3-year rates of aortic-root surgery, aortic dissection, death, and a composite of these events did not differ significantly between the two treatment groups.

\section{CONCLUSIONS}

Among children and young adults with Marfan's syndrome who were randomly assigned to losartan or atenolol, we found no significant difference in the rate of aorticroot dilatation between the two treatment groups over a 3-year period. (Funded by the National Heart, Lung, and Blood Institute and others; ClinicalTrials.gov number, NCT00429364.)
The authors' full names, academic degrees, and affiliations are listed in the Appendix. Address reprint requests to Dr. Lacro at the Department of Cardiology, Boston Children's Hospital, 300 Longwood Ave., Boston, MA 02115-5724, or at ron.lacro@cardio.chboston.org.

*A complete list of investigators in the Pediatric Heart Network is provided in the Supplementary Appendix, available at NEJM.org.

This article was published on November 18, 2014, at NEJM.org.

DOI: 10.1056/NEJMoal404731 Copyright (๑) 2014 Massachusetts Medical Society 
M ARFAN'S SYNDROME IS AN AUTOSOmal dominant disorder of connective tissue affecting approximately 1 in 5000 people. $^{1}$ Cardiovascular disease, mainly progressive aortic-root dilatation and dissection, is the leading cause of death in Marfan's syndrome. After an open-label, randomized trial comparing propranolol with no therapy, published in 1994, showed a reduced rate of aortic enlargement among treated patients, beta-adrenergic receptor antagonists (beta-blockers) became the mainstay of medical management. ${ }^{2}$ Current management includes serial cardiac imaging, exercise restriction, administration of beta-blockers, and elective aortic-root replacement. ${ }^{3}$ Although early diagnosis and refined medical and surgical treatment have improved survival, patients with Marfan's syndrome continue to have high rates of cardiovascular disease and early death.

Marfan's syndrome is caused by mutations in FBN $1,{ }^{4}$ the gene that encodes fibrillin-1, a component of extracellular microfibrils. Fibrillin-1 binds the latent complex of the cytokine transforming growth factor $\beta$ (TGF- $\beta$ ) and regulates its activation and signaling. Studies in a mouse model of Marfan's syndrome showed that a deficiency of fibrillin-1 was associated with excessive signaling by TGF- $\beta$. Excessive TGF- $\beta$ activation and signaling are currently thought to contribute to the pleiotropic manifestations of Marfan's syndrome, including aortic-root dilatation and dissection..$^{5,6}$

The angiotensin II type 1-receptor blocker (ARB) losartan has been shown to attenuate TGF- $\beta$ signaling in some disease states, such as chronic renal failure, presumably by decreasing the expression of TGF- $\beta$ ligands, ${ }^{6,7}$ receptors, ${ }^{8}$ and activators. ${ }^{9}$ The rate of aortic-root growth among mice with Marfan's syndrome treated with losartan was similar to that among wildtype mice and was significantly less than that among untreated littermates with Marfan's syndrome..$^{10}$ Although the beta-blocker propranolol also reduced the rate of aortic-root growth among mice with Marfan's syndrome, this effect was much less pronounced than that seen with losartan. Losartan therapy in mice with Marfan's syndrome prevented elastic-fiber fragmentation and therefore preserved aortic-wall architecture. ${ }^{10}$ Losartan also decreased the rate of aortic-root dilatation in two small series of children with Marfan's syndrome. ${ }^{11,12}$ The goal of the present trial was to determine whether the rate of aorticroot enlargement during treatment with losartan differs from the rate during treatment with atenolol among patients with Marfan's syndrome and a dilated aortic root.

METHODS

\section{STUDY DESIGN}

The study design has been published previously. ${ }^{13}$ The trial was designed and performed by the Marfan Trial Subcommittee of the Pediatric Heart Network (see the Supplementary Appendix, available with the full text of this article at NEJM.org). The protocol (available at NEJM.org) was approved by the institutional review board or ethics committee at each study center. The data were collected by center investigators and analyzed at the data coordinating center (New England Research Institutes). The lead statistician (the third author) vouches for the accuracy of the data and analyses, and all the authors vouch for the fidelity of this report to the trial protocol.

The trial was sponsored by the National Heart, Lung, and Blood Institute, with additional financial support provided by the Food and Drug Administration (FDA) Office of Orphan Products Development and the Marfan Foundation. Merck and Teva Canada provided losartan and atenolol, respectively, but these companies had no role in the design or conduct of the trial, data collection or analysis, or the writing of the manuscript or the decision to submit it for publication.

\section{PARTICIPANTS}

Inclusion criteria were an age of 6 months to 25 years, a diagnosis of Marfan's syndrome according to the original Ghent criteria (Table S1 in the Supplementary Appendix), ${ }^{14}$ and a $z$ score for the maximum aortic-root diameter indexed to bodysurface area (hereafter, aortic-root $\mathrm{z}$ score) greater than 3.0 (as determined by measurement at the local study site). Exclusion criteria were previous or impending aortic surgery; an aortic-root diameter greater than $5 \mathrm{~cm}$; a history of aortic dissection; a diagnosis of the Loeys-Dietz syndrome ${ }^{15}$ or the Shprintzen-Goldberg syndrome ${ }^{16}$; therapeutic rather than prophylactic use of an angiotensin-converting-enzyme inhibitor, beta-blocker, or calcium-channel blocker; prior adverse effects related to treatment with ARB or beta-blocker therapy or a contraindication to such treatment; 
and an inability to complete study procedures. Written informed consent was obtained from each participant or the participant's parent or guardian.

\section{STUDY PROCEDURES}

Participants were assigned to atenolol or losartan in a 1:1 ratio with the use of randomly permuted blocks stratified according to age (young adults [defined as male participants 16 to 25 years of age and female participants 15 to 25 years of age] vs. children [male participants younger than 16 years of age and female participants younger than 15 years of age ] $)^{17}$ and a baseline aortic-root $z$ score of less than 4.5 versus a $z$ score of 4.5 or greater. Dynamic allocation was performed within each center. ${ }^{18}$

Atenolol (at an initial dose of $0.5 \mathrm{mg}$ per kilogram of body weight) was increased on the basis of the hemodynamic response up to a maximum dose of $4.0 \mathrm{mg}$ per kilogram per day (not to exceed a total dose of $250 \mathrm{mg}$ ), with a goal of a $20 \%$ or greater decrease in the mean heart rate as measured on a 24 -hour recording. ${ }^{13}$ Losartan (at an initial dose of $0.4 \mathrm{mg}$ per kilogram) was adjusted on the basis of body weight up to a maximum dose of $1.4 \mathrm{mg}$ per kilogram per day (not to exceed a total dose of $100 \mathrm{mg}$ ), as recommended by the FDA. ${ }^{13}$

Study personnel supervising the dose adjustments were aware of the treatment assignments. All other persons, including participants, families, and other caregivers, were unaware of the treatment assignments. Losartan was provided by the manufacturer as unmarked white tablets. No unmarked tablets were available for atenolol, which was provided by the manufacturer as white tablets that retained the appearance of the commercially available generic agent, so participants and family members may have determined the study-drug assignment. Echocardiographic core laboratory readers were unaware of both the treatment assignment and the study visit number.

\section{FOLLOW-UP AND OUTCOMES}

Study visits occurred at baseline and at 6, 12, 24, and 36 months after randomization. Echocardiograms were obtained at each study visit and were interpreted in a core laboratory. Adherence to the treatment regimen was assessed according to the number of tablets or amount of liquid in the medication bottles that were returned.
The primary outcome was the rate of aorticroot enlargement, expressed as the annual change in the aortic-root $\mathrm{z}$ score, measured by means of echocardiography as previously described, ${ }^{19}$ over the 3-year period after randomization. Secondary outcomes included the rate of change in the absolute diameter of the aortic root; the rate of change in the $z$ score and the absolute diameter of the ascending aorta and the aortic annulus; the rate of change in aortic regurgitation; time to aortic dissection, aortic-root surgery, or death (clinical outcomes); the rate of change in anthropometric outcomes (height, weight, body-mass index, ratio of upper to lower body segment, and ratio of arm span to height); and the incidence of adverse events and participant-reported symptoms.

\section{STATISTICAL ANALYSIS}

We designed the trial to have, with an alpha level of $0.05,85 \%$ power to detect a treatment difference of 0.194 standard-deviation units ( $z$ score units) per year ${ }^{13}$ in the aortic-root $z$ score, after adjustment for treatment crossover, an assumed withdrawal rate of up to $20 \%$, and three interim analyses. The resulting target sample was 604 participants.

The primary analysis was based on the intention-to-treat principle. Secondary analyses were performed with the use of multiple imputation for missing data, as well as with the exclusion of 36 participants who were identified after randomization as having a major eligibility violation, and with adjustment for status with respect to a history of an endocrine disorder, because the prevalence of this baseline characteristic was unequal in the two treatment groups. A nominal $P$ value of less than 0.0453 was required for the two-sided analysis of the primary outcome at the completion of the trial because three interim analyses were performed. Adjustments were not made to the significance levels of hypothesis tests for secondary trial outcomes.

Echocardiographic and anthropometric outcomes were modeled with linear regression with the use of mixed effects to account for the longitudinal design ${ }^{20}$ with the use of compound symmetry and unstructured covariance structures, respectively. The baseline-adjusted rates of change in the two treatment groups were compared with the use of a test of the treatment-bytime interaction effect. Linear regression was 
used to examine the relationship between heart rate and prescribed dose. The time to clinical events according to treatment group and according to subgroup for all the participants was compared with the use of a log-rank test, with event rates estimated according to the KaplanMeier method. The incidence rates of adverse events in the two treatment groups were compared with the use of Poisson regression. Treatment-group comparisons of blood pressure, heart rate, and safety-related laboratory variables were performed with the use of Student's t-test or a Wilcoxon rank-sum test, and treatment-adherence rates and proportions of participants with each reported drug reaction were compared with the use of Fisher's exact test.

The prespecified subgroups defined at baseline were young adults versus children, age as a continuous variable, aortic-root $\mathrm{z}$ score $(<4.5 \mathrm{vs.}$ $\geq 4.5$ ), and previous use of a beta-blocker (yes vs. no). A differential treatment effect according to prespecified subgroup was identified by an interaction test (subgroup by treatment by time) in the longitudinal regression model for the echocardiographic and anthropometric outcomes and in a Cox proportional-hazards regression model for clinical outcomes.

\section{RESULTS}

\section{STUDY PARTICIPANTS}

From January 2007 through February 2011, we enrolled 608 participants at 21 clinical centers; 303 participants were randomly assigned to atenolol and 305 to losartan. The numbers of patients who were screened, randomly assigned to a treatment group, and included in the analysis of the primary outcome are shown in Figure 1. Baseline clinical and echocardiographic characteristics were similar between the atenolol group and the losartan group, except for the incidence of endocrine disorders ( $2 \%$ vs. $0 \%, P=0.007$ ) (Table 1$){ }^{19,21}$ There was no significant difference in the withdrawal rate ( $11 \%$ in each group) or in the median time from randomization to withdrawal (2.3 years in the atenolol group and 1.9 years in the losar$\tan$ group, $\mathrm{P}=0.47$ ).

\section{PRESCRIBED DOSE AND ADHERENCE}

The mean dose of atenolol was $2.7 \pm 1.1 \mathrm{mg}$ per kilogram per day, and the mean dose of losartan was $1.3 \pm 0.2 \mathrm{mg}$ per kilogram per day (Table S2 in the Supplementary Appendix). The mean absolute doses for young adults were $151 \pm 75 \mathrm{mg}$ of atenolol per day and $85 \pm 14 \mathrm{mg}$ of losartan per day. The proportion of patients who returned at least $60 \%$ of medication bottles did not differ significantly between the atenolol group and the losar$\tan$ group (65\% and $64 \%$, respectively; $\mathrm{P}=0.86$ ). On the basis of the amount of medication in the bottles returned, we estimated that $71 \%$ of the participants took at least $80 \%$ of the dispensed dose $(73 \%$ of the participants in the atenolol group and $69 \%$ in the losartan group, $\mathrm{P}=0.30$ ).

\section{BLOOD-PRESSURE AND HEART-RATE EFFECTS}

There were no significant differences between the two treatment groups in baseline blood pressure (Table S3 in the Supplementary Appendix). At 3 years, there was a small but significant difference in the mean diastolic pressure $(54 \pm 8 \mathrm{~mm} \mathrm{Hg}$ in the atenolol group vs. $56 \pm 8 \mathrm{~mm} \mathrm{Hg}$ in the losartan group, $\mathrm{P}=0.04$ ) but no significant differences in the systolic pressure or mean blood pressure (Table S3 in the Supplementary Appendix).

As expected, the resting and average 24-hour heart rates were significantly lower at all study visits in the atenolol group than in the losartan group (Table S3 in the Supplementary Appendix). Among children, higher prescribed doses of atenolol were significantly associated with higher resting and average 24-hour heart rates, suggesting a relative lack of heart-rate responsiveness in some participants during the dose-adjustment process (Fig. S1 in the Supplementary Appendix).

\section{AORTIC-GROWTH OUTCOMES}

The baseline-adjusted annual rate of change $( \pm S E)$ in the aortic-root $z$ score did not differ significantly between the atenolol group and the losartan group $(-0.139 \pm 0.013$ and $-0.107 \pm 0.013$ standard-deviation units per year, respectively; $\mathrm{P}=0.08$ ) (Fig. 2A and Table 2). Both slopes were significantly less than zero, indicating a decrease in the aortic-root $\mathrm{z}$ score in the two treatment groups. There was insufficient evidence to declare a differential treatment effect according to any prespecified subgroup analyses (Fig. S2 and S3 in the Supplementary Appendix), including age as a continuous variable $(\mathrm{P}=0.38$ for interaction) and an alternative definition of young adult versus child ${ }^{13}$ that was based on attainment of maximal height ( $\mathrm{P}=0.38$ for interaction).

A younger age at baseline was associated with 


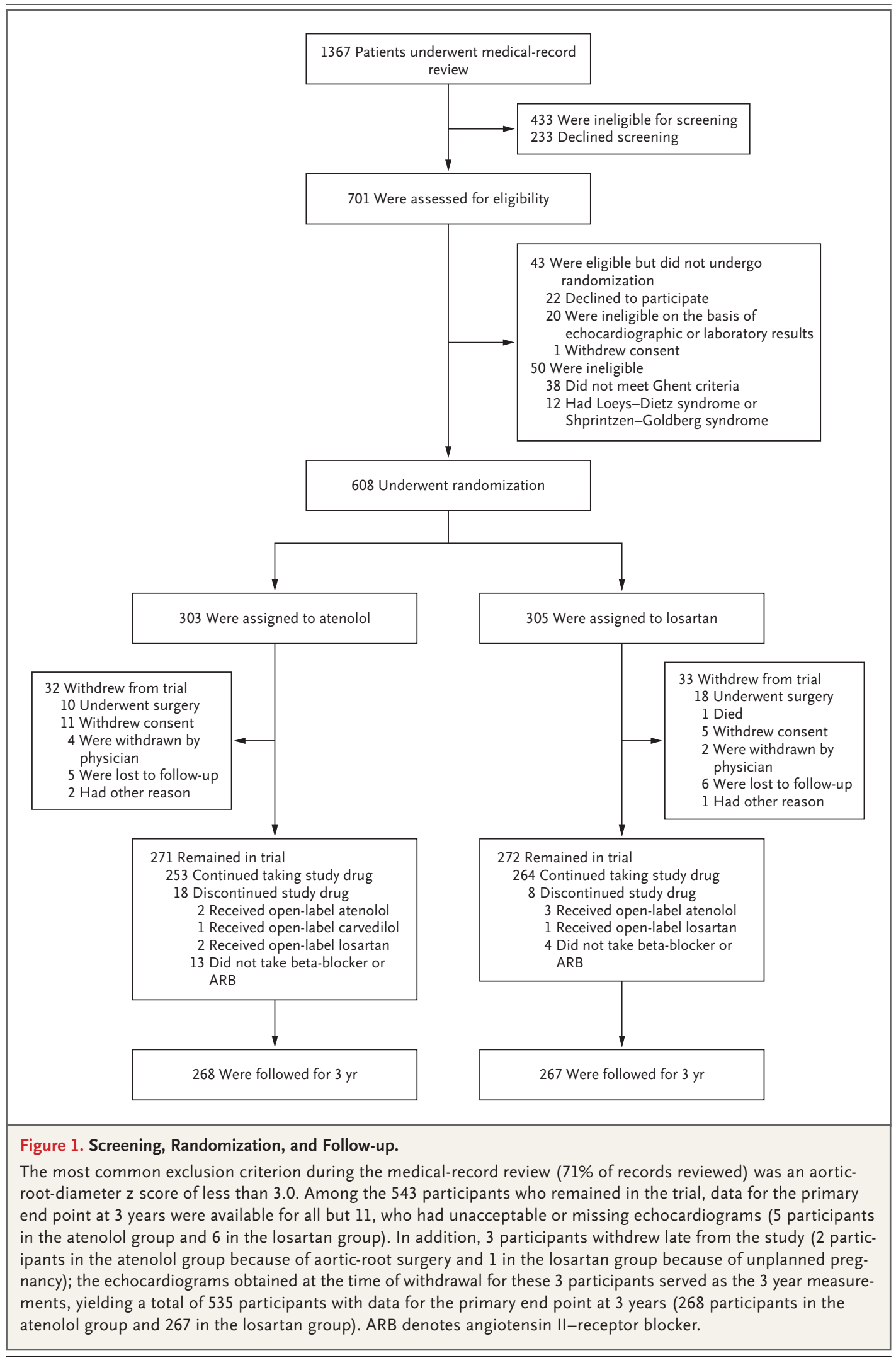

N ENGLJ MED NEJM.ORG

The New England Journal of Medicine

Downloaded from nejm.org by JULIE DE BACKER on November 18, 2014. For personal use only. No other uses without permission. Copyright (C) 2014 Massachusetts Medical Society. All rights reserved. 


\begin{tabular}{|c|c|c|}
\hline Characteristic & $\begin{array}{l}\text { Atenolol } \\
(\mathrm{N}=303)\end{array}$ & $\begin{array}{l}\text { Losartan } \\
(\mathrm{N}=305)\end{array}$ \\
\hline Age $-y r$ & $11.5 \pm 6.5$ & $11.0 \pm 6.2$ \\
\hline Young adult - no. $(\%) \dagger$ & $76(25)$ & $75(25)$ \\
\hline Male sex - no. (\%) & $180(59)$ & $186(61)$ \\
\hline \multicolumn{3}{|l|}{ Race-no. (\%) } \\
\hline White & $266(88)$ & $260(85)$ \\
\hline Black & $21(7)$ & $25(8)$ \\
\hline Asian & $6(2)$ & $10(3)$ \\
\hline Other & $10(3)$ & $10(3)$ \\
\hline Hispanic — no./total no. (\%) $\leftarrow$ & $36 / 302(12)$ & $46 / 305(15)$ \\
\hline \multicolumn{3}{|l|}{ Presence of causal FBN1 mutation - no. (\%) } \\
\hline Yes & $93(31)$ & $88(29)$ \\
\hline No & $9(3)$ & $15(5)$ \\
\hline Unknown & $201(66)$ & $202(66)$ \\
\hline Family history of Marfan's syndrome — no./total no. (\%) & $180 / 295(61)$ & $181 / 290(62)$ \\
\hline \multicolumn{3}{|l|}{ Echocardiographic findings $\rrbracket$} \\
\hline Maximum aortic-root diameter $-\mathrm{cm}$ & $3.4 \pm 0.7$ & $3.4 \pm 0.7$ \\
\hline \multicolumn{3}{|l|}{ Maximum aortic-root-diameter z score } \\
\hline Median & 4.0 & 4.0 \\
\hline Interquartile range & $3.5-4.8$ & $3.3-5.0$ \\
\hline$\geq 4.5$ - no./total no. (\%) & $106 / 303(35)$ & $114 / 304(38)$ \\
\hline \multicolumn{3}{|l|}{ Medical history — no. (\%) } \\
\hline Cardiac surgery & $6(2)$ & $6(2)$ \\
\hline Cardiovascular disorder & $39(13)$ & $36(12)$ \\
\hline Prior use of beta-blocker & $173(57)$ & $171(56)$ \\
\hline Endocrine disorder $\|$ & $7(2)$ & 0 \\
\hline Neurodevelopmental disorder*** & $56(18)$ & $61(20)$ \\
\hline Psychiatric disorder & $23(8)$ & $16(5)$ \\
\hline
\end{tabular}

* Data are adapted from Lacro et al. ${ }^{21}$ Plus-minus values are means \pm SD. The demographic and clinical characteristics did not differ significantly between the two treatment groups ( $P>0.20$ for all comparisons), with the exception of a history of an endocrine disorder $(\mathrm{P}=0.007)$.

$\dagger$ Young adults were defined as male participants who were 16 to 25 years of age and female participants who were 15 to 25 years of age.

$\mp$ Race or ethnic group was reported by the participant or a family member at the time of enrollment.

$\int$ Data are based on readings at a central echocardiographic laboratory. Echocardiographic data were missing for one participant in the losartan group because of an unreadable echocardiogram.

I Cardiovascular disorder was defined by exercise intolerance; syncope; arrhythmia, hypertension, or hypotension requiring therapy; chest pain; shortness of breath; or other cardiovascular conditions.

\| Endocrine disorder was defined by either the use of hormone therapy to limit growth or delayed puberty.

** Neurodevelopmental disorder was defined as attention deficit-hyperactivity disorder requiring therapy, developmental delay requiring therapy, learning disability requiring services, or other neurodevelopmental conditions.

$\uparrow \uparrow$ Psychiatric disorder was defined as depression requiring therapy, anxiety, or other psychiatric conditions.

a greater decrease in the aortic-root $\mathrm{z}$ score over for interaction) (Table S4 in the Supplementary time in the atenolol group $(\mathrm{P}<0.001)$ and in the Appendix). The annual change in the aortic-root losartan group ( $\mathrm{P}=0.002)$, without a significant $\mathrm{z}$ score in children and young adults did not vary difference between the treatment groups $(\mathrm{P}=0.38$ according to the prescribed dose $(\mathrm{P}=0.51$ for 
Figure 2. Change in Aortic-Root z Score and AorticRoot Diameter, According to Treatment Group.

The aortic-root $z$ score is the $z$ score for the maximum diameter of the aortic root, indexed to body-surface area. Panel A shows the baseline-adjusted rate of change in the aortic-root $z$ score over the 3-year period (solid lines), with $95 \%$ confidence intervals (dashed lines) for the pointwise comparison. Panel B shows the baselineadjusted rate of change in the maximum diameter of the aortic root over the 3 -year period (solid lines), with 95\% confidence intervals (dashed lines) for the pointwise comparison.

interaction in the atenolol group; $\mathrm{P}=0.78$ for interaction in the losartan group) (Table S5 in the Supplementary Appendix).

The average annual rate of change in the absolute diameter of the aortic root was similar in the two treatment groups (Table 2 and Fig. 2B). There were small but significant differences favoring atenolol in the average annual change in the absolute diameter and $z$ score for the aortic annulus, but there were no significant differences in the diameter or $\mathrm{z}$ score for the ascending aorta (Table 2). The results of the secondary analyses of the aortic-root $z$ score and data on the anthropometric outcomes are shown in Tables S6, S7, and S8 in the Supplementary Appendix.

\section{ADVERSE CLINICAL OUTCOMES}

The 3-year rates of aortic-root surgery, aortic dissection, death, and a composite of these events did not differ significantly between the two treatment groups (Fig. 3 and Table 2), nor did the treatment effect depend on subgroup. Additional clinical information regarding participants with these events is provided in the Supplementary Appendix. In an analysis that combined the two treatment groups, the 3-year rate of aortic-root surgery was approximately 2 times as high among young adults as among children $(8.5 \%$ vs. $3.8 \%$, $\mathrm{P}=0.03$ ) and approximately 15 times as high among participants with a baseline aortic-root $z$ score of 4.5 or greater as among those with a $z$ score of less than $4.5(12.2 \%$ vs. $0.8 \%, P<0.001)$.

\section{ADVERSE EVENTS AND PARTICIPANT-REPORTED} SYMPTOMS

There was no significant difference between the atenolol group and the losartan group in the overall rate of adverse events (408 events and 365 events, respectively; $\mathrm{P}=0.10$ ) or serious adverse events (40 events and 50 events, respectively; $\mathrm{P}=0.31$ ).

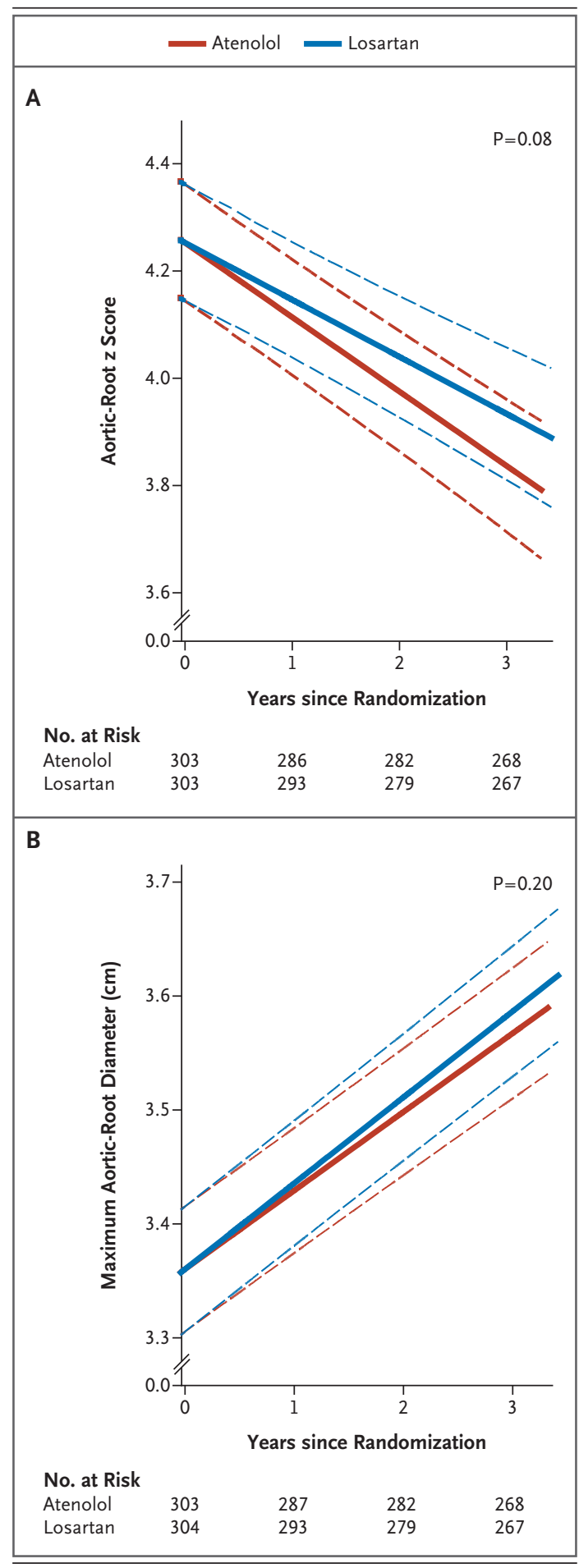

The rate of adverse events that were possibly or probably related to the study drug was higher with atenolol than with losartan (204 events vs. 163 events, $P=0.03$ ), but the results did not differ significantly when the analysis was restricted to 


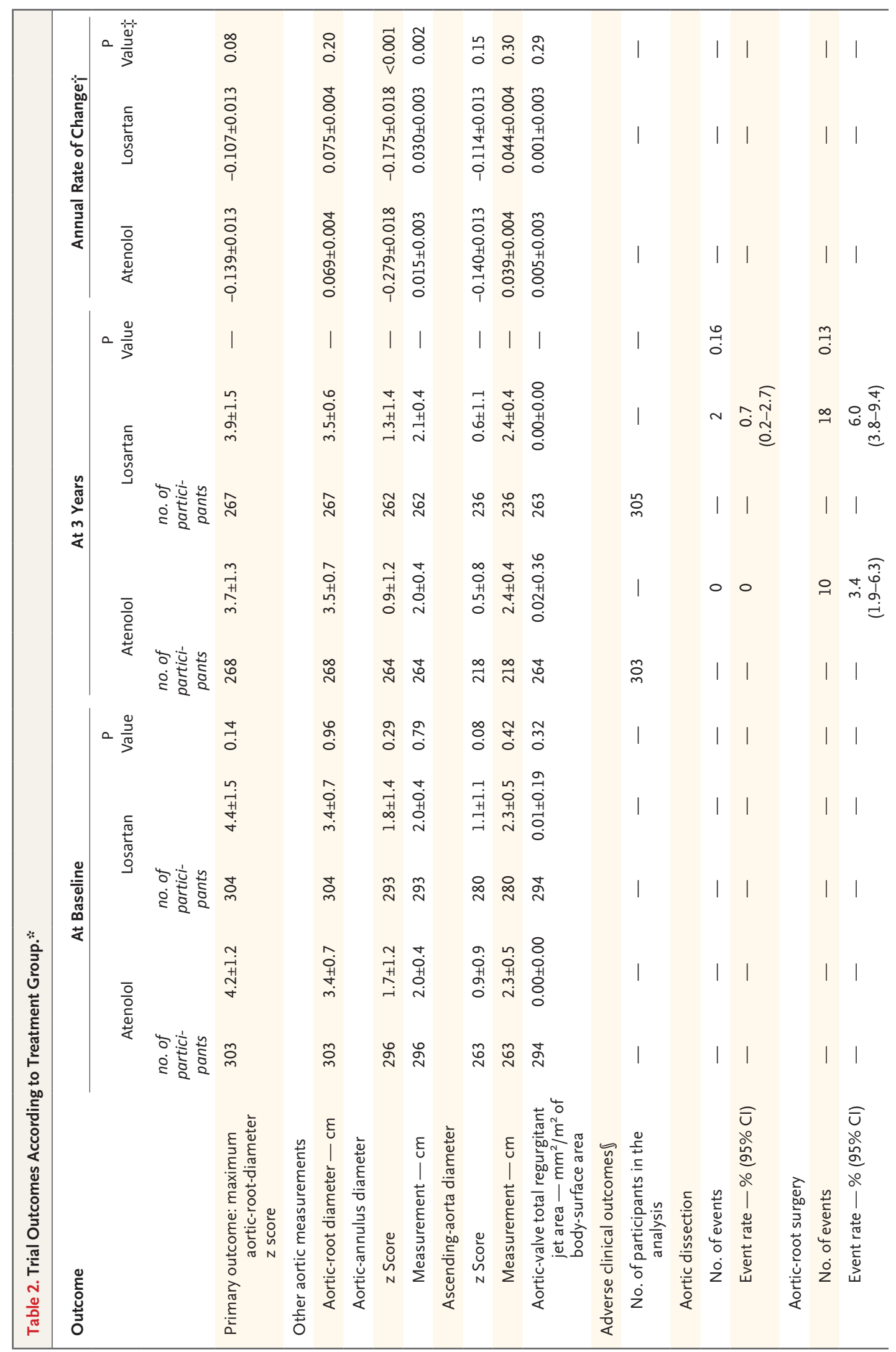




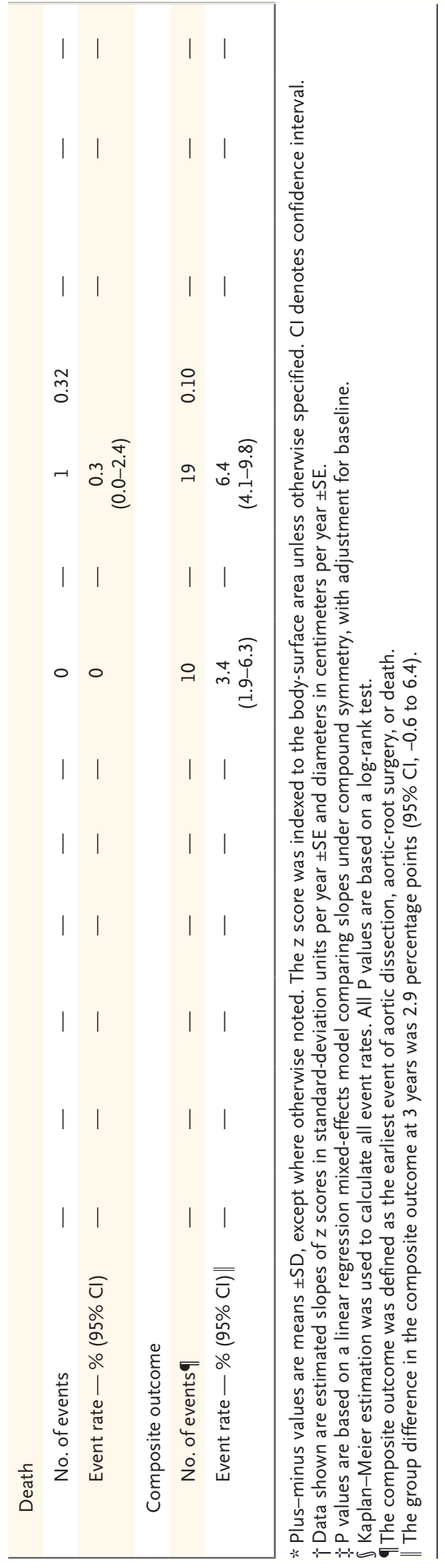

serious adverse events ( 5 events and 2 events, respectively; $\mathrm{P}=0.25)$. Further information about adverse events and patient-reported symptoms is provided in Table S9 in the Supplementary Appendix.

In this randomized trial comparing losartan with atenolol in a large cohort of children and young adults with Marfan's syndrome and a dilated aortic root, we found no significant difference in the rate of aortic-root dilatation, indexed to body-surface area, between the two treatment groups over a 3-year period. The effects of treatment with these agents also did not differ significantly according to prespecified subgroups, including younger versus older participants. We did not find the expected advantage of ARB therapy over beta-blocker therapy. However, we found a small but significant difference in favor of atenolol with regard to the absolute diameter and $z$ score for the aortic annulus. This finding was unexpected, without a clear physiological explanation.

The dose of the beta-blocker used in this study was higher than that used in many other studies. The dose of atenolol was adjusted for a physiological effect, and the rate of change in the aortic-root $\mathrm{z}$ score was not related to dose. The dose of losartan reflected FDA recommendations at the time of protocol development and was in a much narrower range than the dose of atenolol. A higher dose of losartan or a different ARB might have shown a greater effect on aortic growth rate. We also found that diastolic blood pressure was slightly but significantly lower in the atenolol group than in the losartan group, an effect that could reduce the aortic growth rate and perhaps counter the effect of ARB-induced suppression of TGF- $\beta$ signaling in the losartan group.

Although the rate of change in the aortic-root $z$ score did not differ significantly between the two treatment groups, the aortic-root $\mathrm{z}$ score decreased significantly over time in each group, particularly in younger versus older participants, which suggests that there may be value in beginning therapy relatively early in the disease course. Without a placebo group, we are not able to evaluate the magnitude of this benefit, although a previous study comparing beta-block- 


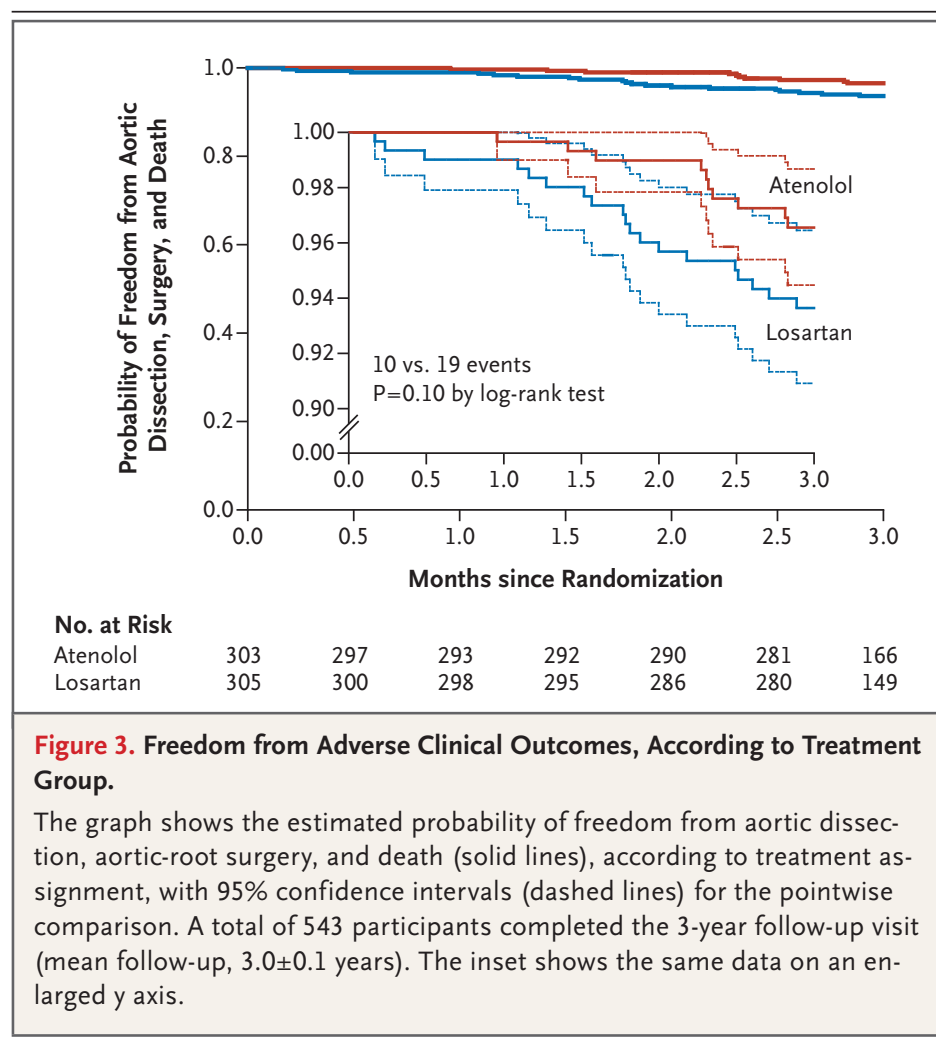

ers with placebo ${ }^{2}$ offers a basis for the conclusion that both agents are effective. The low rate of bothersome participant-reported symptoms is an important finding for both patients and their medical providers because therapy can be selected on the basis of individual patient and provider preference.

There are methodologic differences between our study and other, smaller studies that have shown a benefit of losartan therapy. These include a direct comparison of losartan with a beta-blocker in our study versus comparison of a combination therapy with a beta-blocker alone in other studies, ${ }^{22,23}$ similar blood pressures versus differential lowering in the treatment groups, ${ }^{23}$ the inclusion of both children and young adults versus adults alone, and the exclusion of patients with prior aortic surgery versus the inclusion of such patients. ${ }^{23}$ In one study involving a small number of participants, most of whom were children, combined open-label losartan and betablocker therapy decreased the rate of aortic-root dilatation more than did beta-blocker therapy alone. ${ }^{22}$ The target doses of the beta-blocker administered (maximum dose, $150 \mathrm{mg}$ per day in adults and 2 mg per kilogram per day in chil- dren) were smaller than in our study, and the exact doses achieved were not reported. ${ }^{22}$ In contrast to the patient population in a retrospective study reported by Brooke et al., ${ }^{11}$ which showed a marked beneficial effect of an ARB plus beta-blockers as compared with previous therapy with beta-blockers alone, our study population had a higher average age, the average aortic-root $\mathrm{z}$ score was lower, and the betablocker dose was higher. ${ }^{11}$ Other trials of therapy in patients with Marfan's syndrome are currently under way. ${ }^{24-28}$

This trial had several limitations. First, we did not assess the effect of losartan therapy on TGF- $\beta$. Second, the study results may not be generalizable to persons with Marfan's syndrome who have a body-surface area-adjusted aortic-root $z$ score of 3.0 or less. Third, the statistical power of the study was limited for the detection of significant subgroup findings and treatment differences for relatively low rates of clinical events. Fourth, the primary outcome was assessed by core laboratory personnel who were unaware of the treatment assignments; however, personnel supervising the study-drug adjustments were aware of the treatment assignments, and some participants may have discovered their treatment assignment on the basis of the appearance of the study drug. Finally, we recognize the limitations of a surrogate end point but believed a trial with a hard clinical end point to be impractical, given the rarity of aortic dissection or the need for aortic surgery in young patients.

In conclusion, this trial involving children and young adults with Marfan's syndrome and aorticroot dilatation did not show the expected advantages of losartan over atenolol. The rate of change in the aortic-root $z$ score over the 3-year period did not differ significantly between the atenolol group and the losartan group.

The views expressed in this article are those of the authors and do not represent the official views of the National Heart, Lung, and Blood Institute (NHLBI) or the National Institutes of Health. Supported by U01 grants from the NHLBI (HL068269, HL068270, HL068279, HL068281, HL068285, HL068292, HL068290, HL068288, and HL085057) and the Food and Drug Administration Office of Orphan Products Development and by the Marfan Foundation.

Dr. Dietz reports holding a pending patent $(2014 / 0148,498)$ related to transforming growth factor $\beta$-antagonists, including angiotensin II type 1-receptor blockers, and the multisystem pathogenesis of Marfan's syndrome. No other potential conflict of interest relevant to this article was reported.

Disclosure forms provided by the authors are available with the full text of this article at NEJM.org. 
APPENDIX

The authors' full names and academic degrees are as follows: Ronald V. Lacro, M.D., Harry C. Dietz, M.D., Lynn A. Sleeper, Sc.D., Anji T. Yetman, M.D., Timothy J. Bradley, M.B., Ch.B., Steven D. Colan, M.D., Gail D. Pearson, M.D., Sc.D., E. Seda Selamet Tierney, M.D., Jami C. Levine, M.D., Andrew M. Atz, M.D., D. Woodrow Benson, M.D., Ph.D., Alan C. Braverman, M.D., Shan Chen, M.S., Julie De Backer, M.D., Ph.D., Bruce D. Gelb, M.D., Paul D. Grossfeld, M.D., Gloria L. Klein, M.S., R.D., Wyman W. Lai, M.D., M.P.H., Aimee Liou, M.D., Bart L. Loeys, M.D., Ph.D., Larry W. Markham, M.D., Aaron K. Olson, M.D., Stephen M. Paridon, M.D., Victoria L. Pemberton, R.N., M.S., Mary Ella Pierpont, M.D., Ph.D., Reed E. Pyeritz, M.D., Ph.D., Elizabeth Radojewski, R.N., Mary J. Roman, M.D., Angela M. Sharkey, M.D., Mario P. Stylianou, Ph.D., Stephanie Burns Wechsler, M.D., Luciana T. Young, M.D., and Lynn Mahony, M.D.

The authors' affiliations are as follows: Boston Children's Hospital, Boston (R.V.L., S.D.C., E.S.S.T., J.C.L.); Johns Hopkins University School of Medicine, Baltimore (H.C.D.); New England Research Institutes, Watertown, MA (L.A.S., S.D.C., S.C., G.L.K.); Primary Children's Hospital and the University of Utah, Salt Lake City (A.T.Y.); Hospital for Sick Children, Toronto (T.J.B., E.R.); National Heart, Lung, and Blood Institute, National Institutes of Health, Bethesda, MD (G.D.P., V.L.P., M.P.S.); Medical University of South Carolina, Charleston (A.M.A.); Cincinnati Children's Medical Center, Cincinnati (D.W.B.); Washington University School of Medicine, St. Louis (A.C.B., A.M.S.); Ghent University Hospital, Ghent, Belgium (J.D.B., B.L.L.); Icahn School of Medicine at Mount Sinai (B.D.G.), Children's Hospital of New York (W.W.L.), and Weill Medical College of Cornell University (M.J.R.) — all in New York; Rady Children's Hospital, University of California, San Diego (P.D.G.); Texas Children's Hospital, Houston (A.L.); Vanderbilt University School of Medicine, Nashville (L.W.M.); Seattle Children's Hospital, Seattle (A.K.O.); Children's Hospital of Philadelphia (S.M.P.) and the University of Pennsylvania (R.E.P.), Philadelphia; Children's Hospital and Clinics of Minnesota, Minneapolis (M.E.P.); Duke University Medical Center, Durham, NC (S.B.W.); Ann and Robert H. Lurie Children's Hospital of Chicago, Chicago (L.T.Y.); and the University of Texas Southwestern Medical Center, Dallas (L.M.).

\section{REFERENCES}

1. Judge DP, Dietz HC. Marfan's syndrome. Lancet 2005;366:1965-76.

2. Shores J, Berger KR, Murphy EA, Pyeritz RE. Progression of aortic dilatation and the benefit of long-term beta-adrenergic blockade in Marfan's syndrome. N Engl J Med 1994;330:1335-41.

3. Milewicz DM, Dietz HC, Miller DC. Treatment of aortic disease in patients with Marfan syndrome. Circulation 2005; 111(11):e150-e157.

4. Dietz HC, Cutting GR, Pyeritz RE, et al. Marfan syndrome caused by a recurrent de novo missense mutation in the fibrillin gene. Nature 1991;352:337-9.

5. Dietz HC. TGF-beta in the pathogenesis and prevention of disease: a matter of aneurysmic proportions. J Clin Invest 2010;120:403-7.

6. Lindsay ME, Dietz HC. Lessons on the pathogenesis of aneurysm from heritable conditions. Nature 2011;473:308-16.

7. Everett AD, Tufro-McReddie A, Fisher A, Gomez RA. Angiotensin receptor regulates cardiac hypertrophy and transforming growth factor-beta 1 expression. Hypertension 1994;23:587-92.

8. Fukuda N, Hu WY, Kubo A, et al. Angiotensin II upregulates transforming growth factor-beta type I receptor on rat vascular smooth muscle cells. Am J Hypertens 2000;13:191-8.

9. Naito T, Masaki T, Nikolic-Paterson DJ, Tanji C, Yorioka N, Kohno N. Angiotensin II induces thrombospondin-1 pro duction in human mesangial cells via p38 MAPK and JNK: a mechanism for activation of latent TGF-beta1. Am J Physiol Renal Physiol 2004;286:F278-F287.

10. Habashi JP, Judge DP, Holm TM, et al. Losartan, an AT1 antagonist, prevents aortic aneurysm in a mouse model of Marfan syndrome. Science 2006;312:11721.

11. Brooke BS, Habashi JP, Judge DP, Patel N, Loeys B, Dietz HC III. Angiotensin II blockade and aortic-root dilation in Mar- fan's syndrome. N Engl J Med 2008;358: 2787-95.

12. Pees C, Laccone F, Hagl M, Debrauwer V, Moser E, Michel-Behnke I. Usefulness of losartan on the size of the ascending aorta in an unselected cohort of children, adolescents, and young adults with Marfan syndrome. Am J Cardiol 2013;112: 1477-83.

13. Lacro RV, Dietz HC, Wruck LM, et al. Rationale and design of a randomized clinical trial of beta-blocker therapy (atenolol) versus angiotensin II receptor blocker therapy (losartan) in individuals with Marfan syndrome. Am Heart J 2007; 154:624-31.

14. De Paepe A, Devereux RB, Dietz HC, Hennekam RC, Pyeritz RE. Revised diagnostic criteria for the Marfan syndrome. Am J Med Genet 1996;62:417-26.

15. Loeys BL, Chen J, Neptune ER, et al. A syndrome of altered cardiovascular, craniofacial, neurocognitive and skeletal development caused by mutations in TGFBR 1 or TGFBR2. Nat Genet 2005;37:275-81.

16. Greally MT. Shprintzen-Goldberg syndrome. In: Pagon RA, Adam MP, Ardinger $\mathrm{HH}$, et al., eds. GeneReviews. Seattle: University of Washington, 2006 (http:// www.ncbi.nlm.nih.gov/books/NBK1277). 17. Erkula G, Jones KB, Sponseller PD, Dietz HC, Pyeritz RE. Growth and maturation in Marfan syndrome. Am J Med Genet 2002;109:100-15.

18. Zelen $M$. The randomization and stratification of patients to clinical trials. J Chronic Dis 1974;27:365-75.

19. Selamet Tierney ES, Levine JC, Chen S, et al. Echocardiographic methods, quality review, and measurement accuracy in a randomized multicenter clinical trial of Marfan syndrome. J Am Soc Echocardiogr 2013;26:657-66.

20. Fitzmaurice GM, Laird NM, Ware JH. Applied longitudinal analysis. Hoboken, NJ: John Wiley, 2004.

21. Lacro RV, Guey LT, Dietz HC, et al.
Characteristics of children and young adults with Marfan syndrome and aortic root dilation in a randomized trial comparing atenolol and losartan therapy. Am Heart J 2013;165:828-35.

22. Chiu $\mathrm{HH}$, Wu MH, Wang JK, et al. Losartan added to $\beta$-blockade therapy for aortic root dilation in Marfan syndrome: a randomized, open-label pilot study. Mayo Clin Proc 2013;88:271-6.

23. Groenink M, den Hartog AW, Franken $\mathrm{R}$, et al. Losartan reduces aortic dilatation rate in adults with Marfan syndrome: a randomized controlled trial. Eur Heart J 2013;34:3491-500.

24. Gambarin FI, Favalli V, Serio A, et al. Rationale and design of a trial evaluating the effects of losartan vs. nebivolol vs. the association of both on the progression of aortic root dilation in Marfan syndrome with FBN1 gene mutations. J Cardiovasc Med (Hagerstown) 2009;10:354-62.

25. Detaint D, Aegerter P, Tubach F, et al. Rationale and design of a randomized clinical trial (Marfan Sartan) of angiotensin II receptor blocker therapy versus placebo in individuals with Marfan syndrome. Arch Cardiovasc Dis 2010;103:317-25.

26. Forteza A, Evangelista A, Sánchez V, et al. Study of the efficacy and safety of losartan versus atenolol for aortic dilation in patients with Marfan syndrome. Rev Esp Cardiol 2011;64:492-8. (In Spanish.) 27. Möberg K, De Nobele S, Devos D, et al. The Ghent Marfan Trial - a randomized, double-blind placebo controlled trial with losartan in Marfan patients treated with $\beta$-blockers. Int J Cardiol 2012;157; 354-8.

28. Mullen MJ, Flather MD, Jin XY, et al. A prospective, randomized, placebo-controlled, double-blind, multicenter study of the effects of irbesartan on aortic dilatation in Marfan syndrome (AIMS trial): study protocol. Trials 2013;14:408.

Copyright (c) 2014 Massachusetts Medical Society. 